\title{
Structural basis for the recognition of muramyltripeptide by Helicobacter pylori Csd4, a D,L-carboxypeptidase controlling the helical cell shape
}

\author{
$\underline{\text { Hyerry Jeon }}^{\mathrm{a}}$, Hyoun Sook Kim ${ }^{\mathrm{a}, \mathrm{b}, \mathrm{c}}$, Ha Na $\mathrm{Im}^{\mathrm{a}, \mathrm{b}}$, and Se Won Suh ${ }^{\mathrm{b}}$ \\ ${ }^{a}$ National Cancer Center, Gyeonggi 410-769, ${ }^{b}$ Department of Chemistry, College of Natural Sciences, \\ Seoul National University, Seoul 151-742, ${ }^{c}$ Research Institute of Pharmaceutical Sciences, College of \\ Pharmacy, Seoul National University, Seoul 151-742, Korea
}

Helicobacter pylori infection causes a variety of gastrointestinal diseases, including peptic ulcers and gastric cancer. Its colonization of the gastric mucosa of human stomach is a prerequisite for the survival in the stomach. Colonization depends on its motility, which is facilitated by the helical shape of the bacterium. In H. pylori, crosslinking relaxation or trimming of peptidoglycan muropeptides affects the helical cell shape. Csd4 has been identified as one of the cell shape-determining peptidoglycan hydrolases in H. pylori. It is a $\mathrm{Zn}^{2+}$-dependent D,L-carboxypeptidase that cleaves the bond between the $\gamma$-D-Glu and $m$ DAP of the uncrosslinked muramyltripeptide (muramyl-L-Ala- $\gamma$-DGlu- $m$ DAP) of the peptidoglycan to produce the muramyldipeptide (muramyl-L-Ala- $\gamma$-D-Glu) and $m$ DAP. Here we report the crystal structure of H. pylori Csd4 (HP1075 in strain 26695) in three different states, i.e., the ligand-unbound form, the substrate-bound form, and the product-bound form. H. pylori Csd4 consists of three domains: (i) the N-terminal D,L-carboxypeptidase domain of the typical carboxypeptidase fold, (ii) the central $\beta$-barrel domain of a novel fold, and (iii) the C-terminal immunoglobulin-like domain. The D,L-carboxypeptidase domain recognizes the substrate by interacting primarily with the terminal $m \mathrm{DAP}$ moiety of the muramyltripeptide. It undergoes a significant structural change upon binding either $m$ DAP or the $m$ DAP-containing muramyltripeptide. We also show that Csd5, another cell-shape determinant in H. pylori, is capable of interacting not only with H. pylori Csd4 but also with the dipeptide product of the reaction catalyzed by Csd4. 